\title{
Technology as Social-Material Mediator: From Primary to Secondary Creativity and Beyond
}

\author{
Marina Basu, and Ronald A. Beghetto
}

Arizona State University, Tempe, AZ, USA

\section{ABSTRACT}

How might technology mediate the transition from primary creative expression to secondary creative contributions? In this paper, we address this question by expanding upon recent conceptualizations of primary and secondary creativity (Runco \& Beghetto, 2019) and offer a new way to understand how technology can support creative learning and creative expression. We open by providing a conceptual overview of how technology can serve as a mediator between primary and secondary creativity. We then provide a concrete example of how material artifacts of students' creative expression (primary creativity) were digitized into artifacts, and in turn, transformed again into material creative contributions in the form of narrative volumes (secondary creativity). We also discuss how technology can be used to mediate continuous creative contributions beyond primary and secondary creativity and how creativity researchers can (re)conceptualize the role technology can play in sup-
\end{abstract}

porting indefinite cycles of creative learning and expression from material to digital and back again.

\section{KEYWORDS:}

creative learning, primary and secondary creativity, socio-materiality, technology, continuous creativity

\section{Article history:}

Received: May 14, 2021

Received in revised from: June 20, 2021

Accepted: June 20, 2021

ISSN 2354-0036

DOI: $10.2478 /$ ctra-2021-0002

\section{Corresponding author at:}

Marina Basu

E-MAIL: mbasu3@asu.ed

Ronauld A. Beghetto

E-MAIL: Ronald.Beghetto@asu.edu 
How might technology mediate the transition from primary creative expression to secondary creative contributions? We address this question by drawing on recent conceptualizations of primary and secondary creativity (Runco \& Beghetto, 2019), and offer a fresh perspective on the role technology plays in supporting creative learning and creative expression. We open by providing a conceptual overview of technology as a mediator between primary and secondary creativity. We then provide a concrete example of how student generated material artifacts (primary creativity) were digitized, and in turn, transformed into material creative contributions in the form of narrative volumes (secondary creativity). We will offer new theoretical and empirical considerations for creativity researchers to help them (re)conceptualize the role technology can play in supporting continuous creative learning and expression.

\section{PRIMARY AND SECONDARY CREATIVITY}

The scholarly literature offers various accounts of creative expression, including individual (Runco, 2019) and sociocultural (Glăveanu, 2015). In the former, creativity is typically conceptualized as a personal attribute, while in the latter, creativity is framed within a socio-cultural perspective, wherein the individual and the social are inextricably connected in the creative experiences, processes, and production of artifacts. One recent conceptualization of creativity (Runco \& Beghetto, 2019), called primary and secondary creativity (PSC), endeavored to outline a framework that incorporates the interplay among individual and social-contextual features of creative production. The PSC framework highlights the social, cultural and material influences on the production of creative artifacts. More specifically, according to the PSC framework, primary creativity occurs when an individual (or group), in dialogue with social-material and cultural factors, produces a creative artifact. Secondary creativity takes the primary creative artifact as the starting point of the social creative process, wherein the audience now becomes the creator or interpreter of primary creative outcomes and, in turn, produces secondary creative experiences or artifacts.

The PSC process thus involves two stages, the secondary stage occurring after the primary stage. There is a personal creative act in the first stage, that is taken up by the audience and transformed creatively in the second stage, in which the original creator is no longer involved; however, the creative processes are similar in that there is a dialogue or interaction with the subject matter or material that results in a creative artifact. The PSC model can be depicted in the following iterative (and circuitous) sequence:

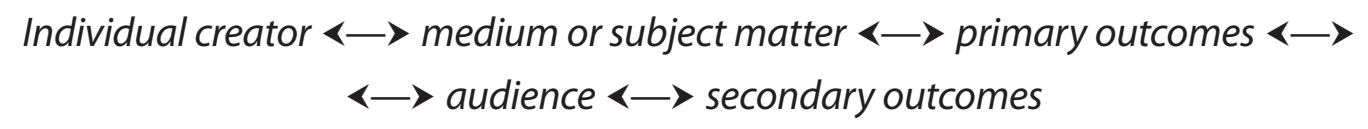

Importantly, creative experiences or artifacts can be considered primary or secondary creativity based on the vantage point or perspective of the people involved. The same creative artifact or 
experience might serve as an example of primary creativity (when viewed from the perspective of the people who are involved in creating the primary artifact) or secondary creativity (when viewed from the vantage point of an audience experiencing or engaging with an artifact). ${ }^{1} \mathrm{~A}$ poem, for instance, can be viewed as primary creativity from the poet's perspective and secondary creativity from reader's perspective who is engaged in appreciating and creatively interpretating the poem.

Introducing technology as a mediator in this framework can open up further possibilities of creative interaction between the primary and secondary components as well as push secondary outcomes into the potentiality of indefinite, continuous creative outcomes. Although there are various ways to conceptualize technology as a social-material mediator, here we draw on ideas of mediation similar to those presented in Wertsch et al. (1995), whereby we assert that digital technologies serve as a bridge or link between the creative actions and experiences of people and their socio-historical environments and settings. When viewed from this perspective, technologies can serve as a means by which creative expression is developed (primary creativity) and continuously experienced through time and space (secondary creativity). Moreover, our perspective recognizes that technologies can afford opportunities to move between material, social, and digital features of our creative experiences and lives.

In this way, primary and secondary creative outcomes can take on an indefinite and inconclusive $(n+1)$ potential for continuous creative outcomes. This enhancement of the primarysecondary creativity framework $\left(\mathrm{PSC}_{\mathrm{n}+1}\right.$ ) highlights how creative sensemaking and the production of creative outcomes can occur indefinitely and aligns with recent dynamic conceptions of creativity (Corazza, 2016) and creative learning (Beghetto \& Vasquez, in press). In the sections that follow, we explore the role of technology and the broader continuum of creative outcomes from primary to secondary and beyond. As we will discuss, the role of technology in creative sensemaking and creative production has been undertheorized especially in educational settings but holds much potential for considering creative learning futures (Beghetto, in press; Henriksen \& Mishra, in press). In general, technology is often discussed in terms of techno-centric or human-centric applications (Boden, 1998). This paper attempts to elaborate on the role of technology in the process of creative production by introducing an integrative approach to conceptualizing technology-human creative interactions.

\section{ROLE OF TECHNOLOGY IN CREATIVITY}

It has been widely asserted and empirically demonstrated that the socio-materiality of environments plays a central role in creative sensemaking and creative production (Glăveanu, 2020; Tanggaard, 2013; Tanggaard \& Beghetto, 2015; Vallée-Tourangeau \& March, 2019). In the context of painting for instance, the paints, the brushes and the canvas shape the final painting as much as the artist's 
imagination. The introduction of technology, particularly digital technologies give rise to the concept of an "immaterial space freed from the physical quality of a material substance" (Giaccardi \& Candy, 2009, p. 194). A stability provided by materialities is replaced by the dynamic and interactive nature of new technologies. This enables a creative "tinkering" or play that digital technologies afford (Giaccardi \& Candy, 2009, p. 195).

A similar idea is found in Treadaway's (2009) discussion of technology and its role in the creative process. Treadway situates her research in the context of textile design and compares physical and digital processes of art creation over the phases of concept generation, conceptual blending, and idea development. She points to the affordances as well as disadvantages of using digital technologies in creating an artwork (see also Glăveanu, 2012). There can, of course, be losses in creative experiences and processes when moving from material to digital technologies. As Treadaway (2009) points out, the friction of a brush on a rough canvas can produce certain sensations that add to the creative process indirectly or directly (for example, sand that blows onto the canvas when drawing a seascape on the beach) that are not possible with digital media. Although sensory details are lost when using digital technologies, digital technologies enable rapid generation of ideas and exploration of different possibilities. Digital technologies thereby provide us with the ability to immediately undo an action and change the outcome and thus allows for more rapid iterations of ideas.

Additionally, digital technologies enable layering and blending of work (integrating digital photographs with other artwork for example) that can otherwise be slow and cumbersome using material objects. Ziegler and Diehl (1998), for instance, demonstrated the potential for computersupported idea generation. Specifically, Ziegler and Diehl found that digital environments may help in idea generation as participants may be more motivated to generate ideas when technology mediates communication and everyone's ideas can be rapidly presented, accessed, and connected. Beyond computational power and immediacy of connections, Treadaway (2009) discusses the increased collaborative potential when using digital technologies to create something and, most germane to our present argument, technologies can help mediate and distribute creative experiences and artifacts among people in different geographical and temporal locations. Indeed, as Henriksen, Mishra and Fisser (2016) note, "ease of creation and ease of sharing" are two affordances that are specifically provided by technology (p. 35). It is this very nature of technology to maintain creative contributions beyond particular spatial and temporal boundaries that allows it to serve as a powerful mediator for continuous creative contributions (Beghetto, in press).

With the above discussion in mind, we now explore the process of technology mediated creative process in detail with the help of a specific empirical example. Returning to our discussion of the $\mathrm{PSC}_{\mathrm{n}+1}$ framework, we can recognize how technology can mediate various points along the iterative primary, secondary, and continuous creative process (again recognizing that although the process is often sequential, it is not always linear or stepwise). Specifically, technology can be leveraged as a potential mediator between any components of the PSC $n+1$ sequence. Technology can, for instance, be used to mediate the primary phase of creative engagement and sensemaking, 
the production of primary outcomes, the creative sensemaking of secondary audiences, and mediate the production and audience engagement with secondary outcomes and continuous creative outcomes. Introducing technology as a mediator in the framework of primary and secondary creativity can open up multiple possibilities of creative interaction, engagement, and production of creative outcomes. Technology can also offer multiple sensory, imaginative, and enacted possibilities during the act of creative expression and in our experiences of the creative expression of others, which may not otherwise be available. Students, for example, can use technology to interact with paintings or other forms of creative expressions from different perspectives, which, in turn, can help promote new ways of thinking, acting, and creating for those students and others in and beyond the classroom. ${ }^{2}$ This is because technologies afford us opportunities to experience phenomena from new and different perspectives across time and space.

We now turn our attention to the discussion of an empirical case, which illustrates the PSC $n+1$ model in action and how the process features socio-material, digital technology, and human entanglements. Specifically, we highlight how technology was used to move from material to digital and back to material creative artifacts, mediating the transition between primary creative outcomes and secondary creative production. We also discuss how technology can be used to move from secondary creative outcomes to continuous creative outcomes. This relationship is illustrated in Figure 1.

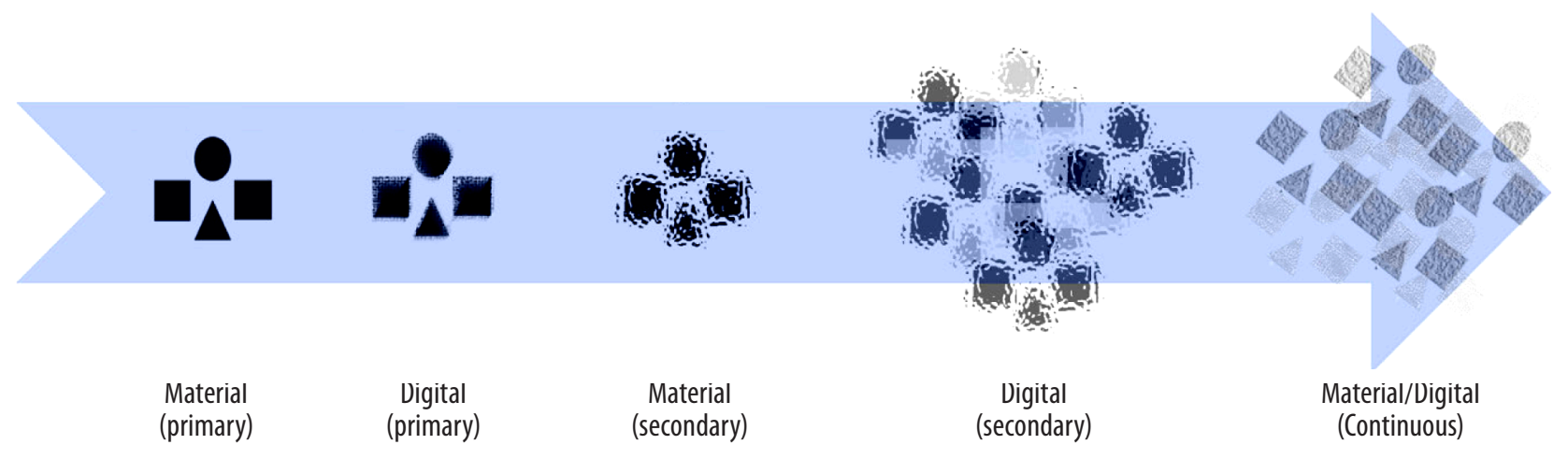

Figure 1. From Material to Digital and Beyond

As illustrated in Figure 1, material creative outcomes from primary creative work can be transformed through technology into a digital medium that, in turn, can be worked with and developed into secondary material outcomes. These secondary outcomes can also be digitized and thereby serve as the potential for continuous, on-going creative work and outcomes. In what follows we provide a brief description of the context of our empirical example and then go on to discuss how the assemblage of materials, digital technologies, and creative engagement produced primary, secondary, and potentially continuous creative outcomes.

2 We want to thank an anonymous reviewer for highlighting this example illustrating the unique affordances of technology when viewed in light of primary, secondary, and continuous creative experiences. 


\section{AN EXAMPLE OF TECHNOLOGY MEDIATED PSC $\mathbf{C}_{\mathbf{N}+1}$}

\section{Creative Conducive Context}

Marina, the first author of this manuscript, taught the elementary grades for several years in India, in a non-traditional school where there was a strong focus on arts and creativity. Basu (in press) provides more context about the curricular activities and the educational philosophy of the school. In keeping with the school's philosophy, all students and teachers were encouraged to engage in creative activities; teachers were encouraged and supported to teach creatively as well. The art curriculum focused on open ended creative inquiry, exploring various art forms (wood working, painting, weaving, among others) and learning directly from artists and artisans. To meet these objectives, artisans and craftspeople were often invited to the school campus to conduct workshops with children on specific traditional art forms.

In accordance with the organizational structure of the school, the art curriculum was tailored to meet the needs of the three different age groups of students in the school. The younger children learned the basics of multiple art forms, the middle schoolers were exposed to certain art forms in greater depth, and the older students could select a particular art form that they wanted to focus on exclusively. The school would also have an annual exhibition, where all the artwork of students and teachers would be on display for parents and other visitors to the school. The school thus provided a rich set of creative experiences for students, teachers, families, and visitors. In what follows, we focus on a specific example of how this context, coupled with digital technology illustrates the process of moving from primary to secondary and continuous creativity.

\section{Case Example}

One particular year at the school, traditional artists were invited to conduct a workshop on Gond painting for the middle school children. Gond painting originated in central India and is characterized by its use of bold colors and simple shapes to depict animals, plants and humans. After being introduced to this art form, the middle schoolers were encouraged to draw and paint using a similar style; the subject matter was their own choice. Most children painted pictures of animals in vivid colors. The paintings had been left to dry near the art classroom - and it was here that Marina was walking by and the paintings caught her eye.

Marina: The colours were almost pulsating - they grabbed my attention. The paintings were lively and I wondered if I could build a narrative out of some of the paintings. The art teacher had taken photographs of all the paintings and shared the digitized versions with me, suggesting that I could create some picture story books for children for the upcoming annual exhibition. That evening, as I was looking at the thumbnails of all the paintings in the digital folder, more than a hundred of them, the vibrant colors - particularly the reds and blues (among other colors) - grabbed my attention. I started sorting the painting based on the dominant color in each, creating two digital folders for the two colors. Digitization enabled me to take children's paintings - the primary creative artifacts 
- and arrange and rearrange them according to the colors that were dominant in each. Some of the digital copies of the paintings in these two folders ultimately led to the creation of two different story books. While the stories have specific titles, I referred to them during the process of creation (and continue to do so even today) as "the red book" and "the blue book."

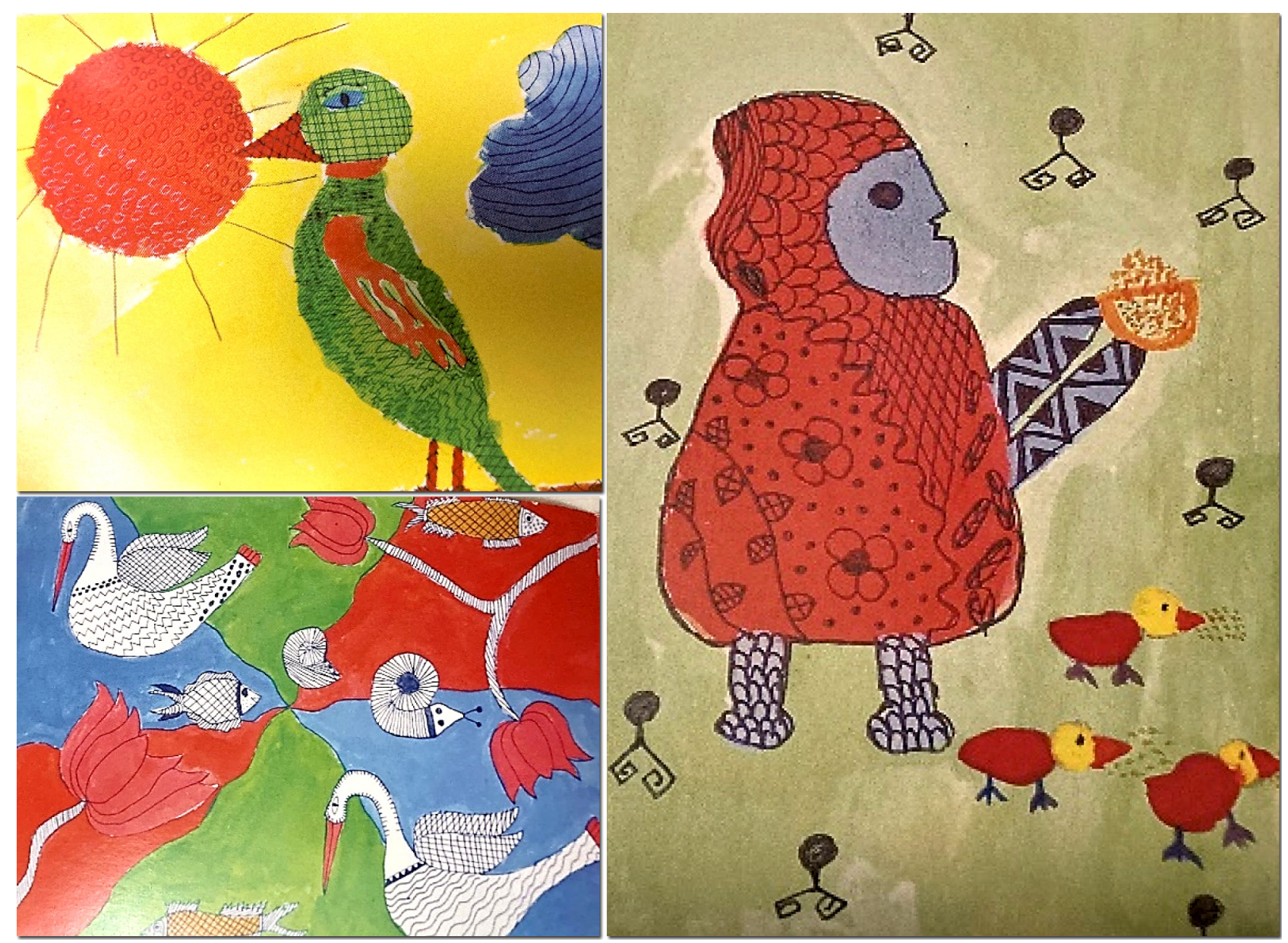

Figure 2. Example Images From the "Red Book"

Examples of the images in the red book included: a red lake, a bird with a red beak, a red apple, the red in a peacock's eye, a child wearing red clothes, a red tree - images that were unrelated to each other, as they were painted by different students (see Figure 2). However, as I was looking through the images in the folder, connections emerged between them, and a narrative began to take shape. Using simple presentation software, I started sequencing them in a certain order and added text to the images. The final story book design involved playing around with some of the images - cropping parts of an image to use as a page border or as the watermarked background to the text. The "red book" transformed into a story about the sun.

The blue book included images of a dark blue sky, blue feathers of a peacock, a leopard with blue spots - and another story began emerging from the pictures. This turned out to be a story about the night when there was no moon in the sky. Designing the book involved more playing with cropped and rotated images, using mirrored images to create special effects, and other tinkerings with the 
technology. Creating copies of images and editing them using digital image editing software helped design certain pages. For example, the cover design for one of the books (see Figure 3) was facilitated by the use of technology. This particular drawing of the clouds was taken from yet another category of images, the black and white paintings that led to yet another story, what I referred to as the "black and white" book, but was here used in creating the front cover of the "blue book."

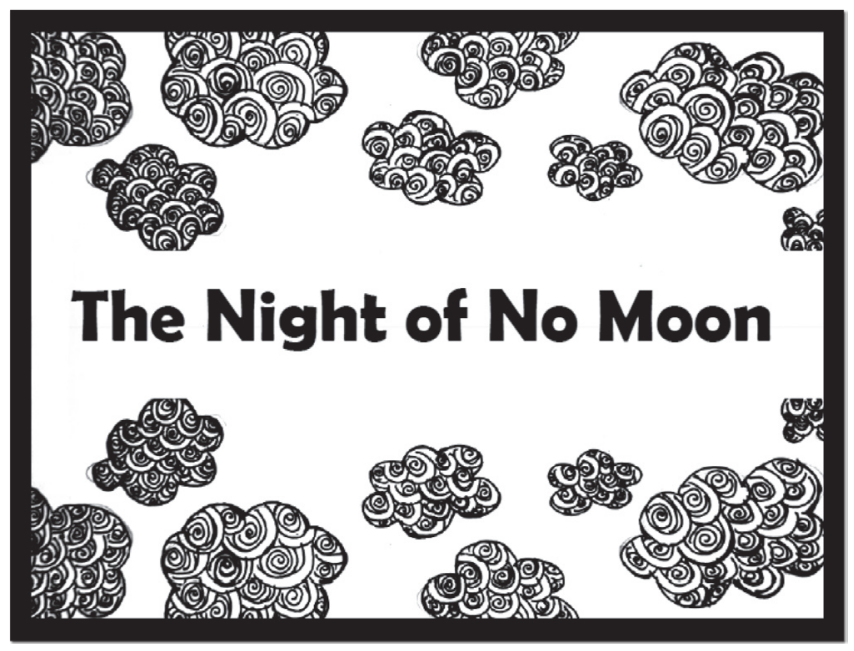

Figure 3. Book cover designed using cropped and mirrored image

It is important to note here that the paintings were creative products in themselves; they were not created following any given theme or keeping in mind the possibility of a story and they are thus the primary creative artifacts. Children explored color and form in the context of the Gond painting workshop and emulated the colors and drawing styles of Gond artists. However, it is worth noting that, from the perspective of the Gond artists, the Gond paintings would be the primary creative artifacts whereas children's paintings would represent secondary creative artifacts.

A child had painted a snake, lying coiled up. It seemed to be a necessary character in both stories, and so I played around with the colour settings to create two differently coloured versions of the same image (see Figures $4-a$ and $b$ ).
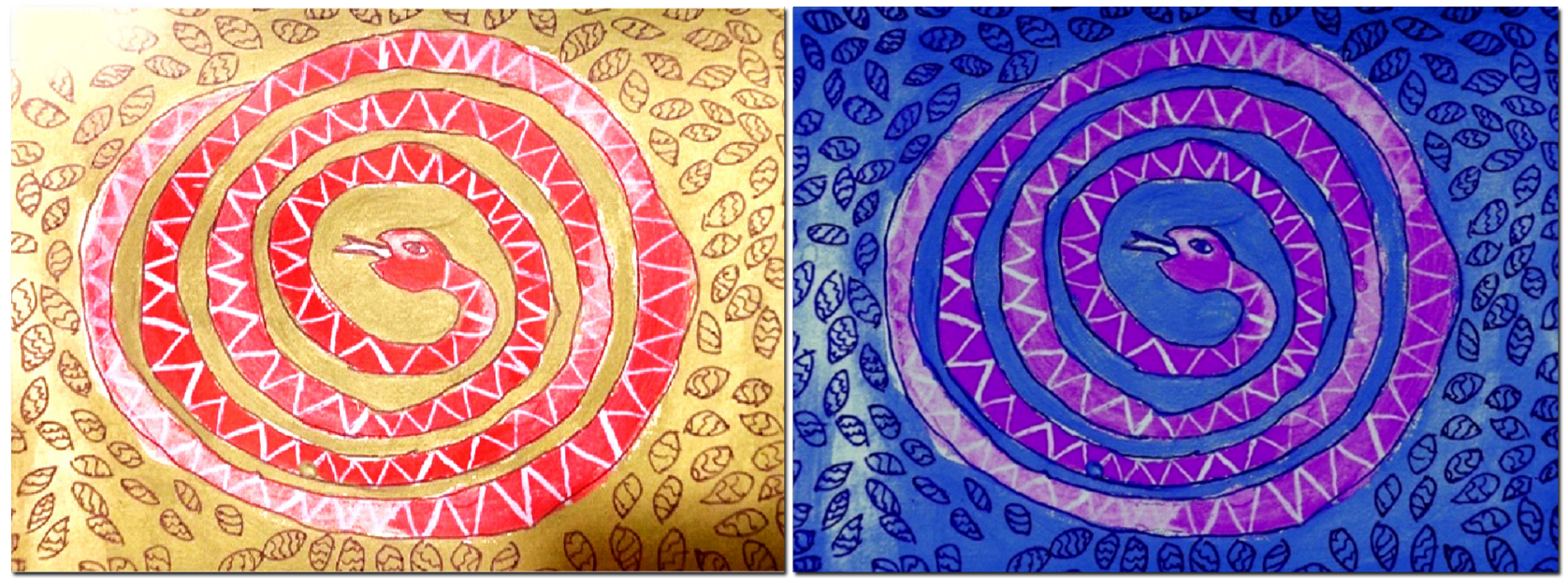

Figures 4 ( $a$ and $b$ ). Examples of recoloring an image with software 
Each version of the snake's image seemed to exude a certain character, and they became a part of both the stories. Since I was working with digital copies, it was easy to reuse a painting for multiple purposes in different books. This was an affordance uniquely provided by digital media. Had I been working with the physical copies of the paintings, I would not have been able to generate a differently coloured version of the same painting in the blink of an eye. The entire process itself would have been quite time consuming if I were to work with the original paintings on paper. There would be the need to handle them delicately, making sure they did not get damaged. Sorting the hundred odd paintings manually might have seemed daunting in the limited time I had beyond my regular teaching responsibilities. Also, sorting them according to color might not even have arisen as a possibility in the first place, had I been looking at a sheaf of paintings.

\section{The Role of Technology in Mediating Primary, Secondary and Continuous Creativity}

We can now consider how this empirical example illustrates core features of the role that digital technology can play in mediating primary, secondary and continuous creativity $\left(\mathrm{PSC}_{\mathrm{n}+1}\right)$. Recall that primary creativity commences with a person's (or group's) engagement with some material or conceptual medium. In this case, students engaged in both primary and secondary creativity (Runco \& Beghetto, 2019) through the process of experiencing and developing their own unique representations of Gond paintings. This is also an example of creative learning (Beghetto, in press), because students were introduced to the creative learning stimulus of Gond painting through a workshop and were invited to engage in their own creative sensemaking process of producing their own Gond paintings.

Although the visiting artist and students did not use digital technologies in the production of the primary creative outcome of student-generated Gond paintings, technologies could have played a role in this phase both in the case of introducing the Gond painting learning stimulus to students and in the production of digital Gond paintings. Doing so, however, may have produced a different creative experiential trajectory. Indeed, it is possible that Marina would have not been inspired to engage with the students' paintings as a stimulus for secondary creativity if they were developed and ensconced in digital devices rather than produced with physical materials and displayed in the physical surrounds of the school environment. We mention this "analog" aspect of the process to highlight the recognition that the use of digital technologies simultaneously offer and deny particular creative affordances (Treadaway, 2009) when it comes to a full trajectory of creative learning expression possibilities, such as those outlined in the $\mathrm{PSC}_{\mathrm{n}+1}$ framework.

The analog display of students' primary creativity is what caught the eye of Marina and it was through this engagement with the creative work that Marina was inspired to use the digitized images as a stimulus for her own secondary creativity. It was at this point that technology served as a mediator between the initial engagement of the students' primary creative outcomes and Marina's secondary creative expression and production of two, material story books. Digital technologies played a role in mediating the initial "concept generation" (Treadaway, 2009), facilitating fluid ideational generation and arrangement of ideas and images (Zeigler \& Diehl, 1998). 
More specifically, digitizing the images and working with them facilitated rapid sorting into reds and blues as well as facilitated sequencing and rearranging the images in various ways, that may have not been possible when working with the physical paintings themselves.

Moreover, picture story books usually start with a storyline or at least the seed of an idea, for which the illustrator then creates specific illustrations. Often, the illustrator is working with the completed story and drawing scenes to match the story's highlights. The process was reversed here: the images shaped a certain story - the story emerged from the paintings. This emergent shaping of creative work has been described elsewhere as a necessary feature of creative production (Anderson, 1987). The horizon of possibilities of an artist making a painting, for instance, is necessarily limited with each subsequent brush stroke - this is how creative engagement moves from an indefinite horizon of possibilities into more (at least temporarily) stabilized point of creative resolution (Beghetto, 2019).

Marina used technology to creatively blend various combinations of elements in an effort to develop new outcomes. Technology served as a means for cropping, duplicating, mirroring, rotating, juxtaposing, and playing with different combinations of images and text. In this way, technology can help facilitate the creative combinatorial aspect of creative work (Rothenberg, 2014; Treadaway, 2009). The ease of moving between different combinations offered flexibility (Zeigler \& Diehl, 1998) in the generation of ideas in different categories; which further led to originality - a unique story idea was generated; and elaboration - a story book was created with fully developed ideas based on the images. Idea generation was facilitated by the easy access to multiple digital images, helping draw connections between images and thus leading to a creative act. Idea development was also facilitated by the use of digital tools. Once compiled, the books were printed and published into a material form that could be shared with others - moving from digital back to material. Moreover, because the books are also in digital form, they can serve as a stimulus for continuous creative contributions, including inspiring new creative works based on the stories or new ideas that can inform the field of creativity studies itself (as is the aim of this paper).

\section{CONCLUDING THOUGHTS}

In this brief article, we have attempted to highlight how technology mediates movement between primary, secondary, and continuous creativity. In this way, we have elaborated on the PSC model by noting how technology adds continuous creative possibilities to move from primary to secondary and continuous creative engagements and outcomes ( $\mathrm{PSC}_{n+1}$ ). Although we have focused on how technology can facilitate creativity by mediating the transition between primary and secondary creativity, we want to also highlight the material and human factors as well. In this particular case, the stories might not have emerged had Marina not been engaged with stories in myriad ways for several years. Marina had grown up reading well-written and beautifully illustrated books; her graduate studies and courses in children's literature had helped her appreciate children's books in 
ways that she had not thought of as a child reader; and finally, in creating the books, she was also drawing upon implicit domain knowledge of story elements and the skills of storytelling, as she had taught language arts for several years.

As has been mentioned, it is also important to keep in mind that in addition to technology facilitating creativity, technology might also impede or hinder creativity. Digital art works remove texture, a feature that is so important to artists as Treadaway (2009) points out. In the context of children's story books, authors who are also illustrators can play with form and content in a way that Marina's work through technology can never approximate. However, technology adds to the process of creativity in unique ways when the thinking shifts from the focus on an individual creator and individual creative outcomes to theorizations of primary, secondary, and continuous creativity $\left(\right.$ PSC $\left._{n+1}\right)$. Moreover, although our case-study focused on creative expression in the arts, it is important to note that technological affordances are not limited to the arts. Technologies can also enhance (or suppress) creative expression in almost any creative endeavor. Exploring these affordances and limitations of technologically mediated creative experiences in and beyond the arts is thereby an important area of continued examination for creativity researchers and practitioners.

Another important consideration is the role of time in creativity. Sternberg (2006) points to the need for slowness in incubating and developing creative potential, something that is removed in the rapidity that comes with technological tools. On the other hand, creativity can also flourish with enabling constraints (Stokes, 2010) and as the above empirical example shows, the movement from primary to secondary creativity was facilitated by the speed afforded by technology, as Marina only had limited time at her disposal.

Taken together, we hope the ideas presented in this short article help inspire creativity researchers to further their own exploration of the role that technology might play in supporting (and potentially impeding) creative production, particularly as it relates to movement from primary material creative outcomes to digital secondary and continuous creative engagement and production ( $\mathrm{PSC}_{\mathrm{n}+1}$ ). We encourage researchers to also consider how technology might play a facilitating (or impeding) role throughout various points of creative engagement and creative production, including considering the entanglements of the socio-material, cultural, and temporal features of creative experiences and endeavors.

\section{References}

Anderson, D.R. (1987). Creativity and the philosophy of C.S. Peirce. Hingham, MA: Kluwer.

Basu, M. (in press). Krishnamurti's Insights on Observation for Global Childhood Education and Research. In N.J. Yelland, L. Peters, N. Fairchild, M. Tesar \& M.S. Perez (Eds), The SAGE Handbook of Global Childhoods (pp. 219-233). Sage.

Beghetto, R.A. (2019). Abductive reasoning and the genesis of new ideas: Charles S. Peirce. In V. P. Glaveanu (Ed.), Creativity Reader. Oxford, UK: Oxford University Press.

Beghetto, R.A. (in press). Engaging uncertainty: Principles and provocations for promoting creative learning futures. In D. Henriksen \& P. Mishra (Eds.), Creative provocations: Speculations on the future of creativity, technology \& learning. Switzerland: Springer. 
Beghetto, R.A., \& Vasquez, A.M. (in press). Creative learning: A pedagogical perspective. In L.J. Ball \& F. Vallee-Tourangeau (Eds.), Routledge International Handbook of Creative Cognition. New York: Routledge

Boden, M. (1998). Computer Models of Creativity. In R. Sternberg (Ed.), Handbook of Creativity (pp. 351-372). Cambridge: Cambridge University Press. https://doi.org/10.1017/CBO9780511807916.020

Corazza, G.E. (2016). Potential originality and effectiveness: The dynamic definition of creativity. Creativity Research Journal, 28, 258-267.

Giaccardi, E., \& Candy, L. (2009). Creativity and cognition 2007: Materialities of Creativity. Leonardo, 42(3), 194-196.

Glăveanu, V.P. (2012), What can be done with an egg? Creativity, material objects, and the theory of affordances. Journal of Creative Behavior, 46(3), 192-208. https://doi.org/10.1002/jocb.13

Glăveanu, V.P. (2015). The status of the social in creativity studies and the pitfalls of dichotomic thinking. Creativity. Theories - Research - Applications, 2, 102-119. https://doi.org/10.1515/ctra-2015-0016

Henriksen, D., \& P. Mishra (Eds.). (in press). Creative provocations: Speculations on the future of creativity, technology \& learning. Switzerland: Springer.

Henriksen, D., Mishra, P., \& Fisser, P. (2016). Infusing creativity and technology in 21 st century education: A systemic view for change. Journal of Educational Technology \& Society, 19(3), 27-37.

Rothenberg, A. (2014). Flight from wonder: An investigation of scientific creativity. New York, NY: Oxford University Press.

Runco M.A. (2019) Creativity as a Dynamic, Personal, Parsimonious Process. In: Beghetto R., Corazza G. (Eds.), Dynamic perspectives on creativity. Creativity theory and action in education, vol. 4. Springer, Cham. https://doi.org/10.1007/978-3-319-99163-4_10

Runco, M.A. \& Beghetto, R.A. (2018). Primary and secondary creativity. Behavioral Sciences, 27, 7-10.

Sternberg, R.J.(2006). The nature of creativity. Creativity Research Journal, 18(1), 87-98. http://dx.doi. org/10.1207/s15326934crj1801_10

Stokes, P.D. (2010). Using constraints to develop creativity in the classroom. In R.A. Beghetto \& J.C. Kaufman (Eds.), Nurturing creativity in the classroom (pp. 88-112). Cambridge University Press.

Tanggaard, L. (2013). The sociomateriality of creativity in everyday life. Culture \& Psychology, 19, 20-32. https://doi.org/10.1177/1354067X12464987

Tanggaard, L., \& Beghetto, R.A. (2015). Ideational pathways: Toward a new approach for studying the life of ideas. Creativity: Theories - Research - Applications, 2, 129-144. https://doi.org/10.1515/ctra-2015-0017

Treadaway, C. (2009). Materiality, memory and imagination: Using empathy to research creativity. Leonardo, 42(3), 231-203.

Vallée-Tourangeau, F., \& March, P.L. (2019). Insight Out: Making Creativity Visible. Journal of Creative Behavior, 54, 824-842. https://doi.org/10.1002/jocb.409

Wertsch, J.V., Del Rio, P., Alvarez, A. (1995). Sociocultural studies of mind. New York: Cambridge University Press.

Ziegler, R., \& Diehl, M. (1998). Computer supported idea generation. In R. Sternberg (Ed.), Handbook of Creativity (pp. 351-372). Cambridge: Cambridge University Press. https://doi.org/10.1017/CBO9780511807916

(c) Copyright by Faculty of Education, University of Bialystok,

20 Swierkowa St., 15-328 Bialystok, Poland

tel. +48857457283

e-mail: creativity@uwb.edu.pl

http://www.creativity.uwb.edu.pl 